\title{
The ATLAS Tracker Upgrade: Short Strip Detectors for the SLHC
}

\author{
Urmila Soldevila on behalf of the ATLAS SCT Collaboration \\ 7 October 2009
}

11th ICATPP Conference on Astroparticle, Space Physics, Detectors and Medical Physics Applications Villa Olmo (Como, Italia) 5-9 October 2009 


\section{Outline}

$>$ LHC vs. SLHC

$>$ Inner Detector (ID)

$>$ Upgrades to the ATLAS Inner Detector

$>$ Upgrade ID Layout

$>$ Radiation Hard Technologies: n-on-p

- Miniature sensors:

- Charge Collection under neutron and proton irradiation

- Full Depletion Voltage

- Full size prototypes sensors:

- Electrical Characterization

$>$ Module Integration Concepts

- Barrel Stave

- End-Cap Stave (Petal)

- Super-Module

$>$ Conclusions 


\section{LHC vs. SLHC}

\section{LHC}

$>$ Designed for luminosity of $10^{34} \mathrm{~cm}^{-2} \mathrm{~s}^{-1}$

$>$ In the first 5 years $700 \mathrm{fb}^{-1}$ integrated luminosity

$>$ First Colliding beams in November 2009

\section{Super-LHC (SLHC)}

$>$ Designed for luminosity of $10^{35} \mathrm{~cm}^{-2} \mathrm{~s}^{-1}$

$>$ SLHC Upgrade plans envision $3000 \mathrm{fb}^{-1}$ (5 years)

$>$ Starting around 2018-2020 


\section{Inner Detector (ID)}

$\underline{\text { Inner Detector }} \longrightarrow$ Vertex (pixel) + Tracker (Silicon Strips, TRT)

High Occupancy :

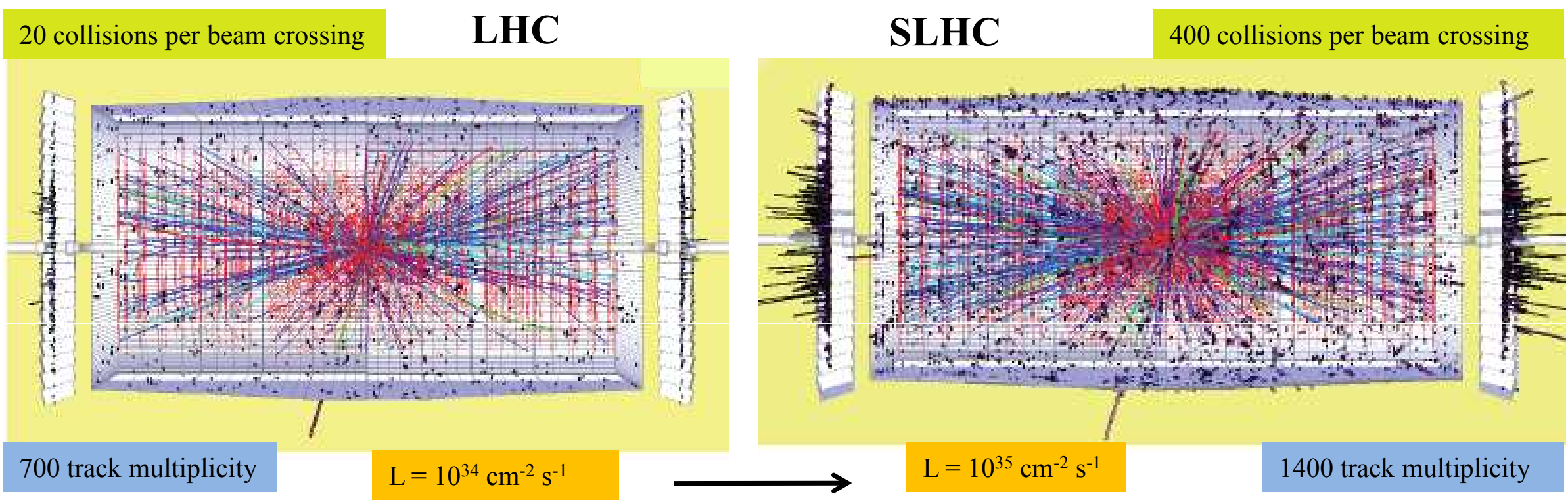

$>$ TRT unable to cope with SLHC occupancy $\rightarrow$ All Silicon ID

$>$ Finer granularity for the detectors to keep the occupancy at the same level of LHC

$>$ This supposes for services:

- x5 number of channels (for SCT)

- In the same space !! 


\section{Inner Detector (ID)}

\section{Particle Fluence :}

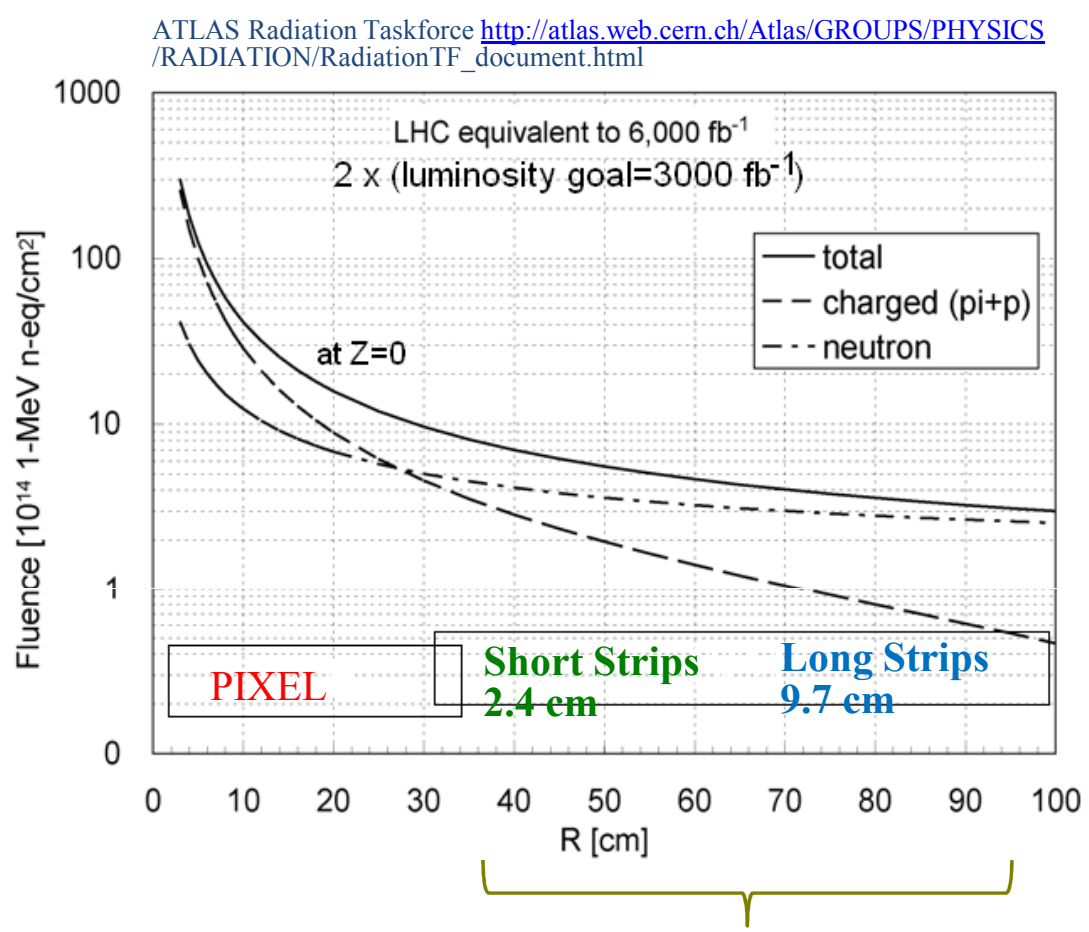

Neutrons $>50 \%$ at $\mathrm{R} \geq 25 \mathrm{~cm}$

$>$ Strip detector damage largely due to neutrons

Designed fluences for sensors:

- B-layer at $3.7 \mathrm{~cm}: 2.2 \times 10^{16} 1 \mathrm{Mev}$ n-equivalent $/ \mathrm{cm}^{2}$

- Outer pixel layers: $3 \times 10^{15} 1 \mathrm{Mev}$ n-equivalent $/ \mathrm{cm}^{2}$

- Middle strip layer at $38 \mathrm{~cm}: 10^{15} 1 \mathrm{Mev}$ n-equivalent $/ \mathrm{cm}^{2}$

- Outer strip layer at $95 \mathrm{~cm}: 4 \times 10^{14} 1 \mathrm{Mev}$ n-equivalent $/ \mathrm{cm}^{2}$

Barrel SCT Upgrade : $38<\mathrm{R}<95 \mathrm{~cm}$

This implies higher radiation hardness for sensors

The ID will be replaced and technologically improved $\rightarrow$ R\&D in RD50 


\section{Upgrades to the ATLAS Inner Detector}

$>$ New Detector Layout: Only silicon pixel and strips.

$>$ Radiation hard technologies: n-on-p silicon strips.

$>$ New Module Integration Concepts (low material budget).

$>$ New Powering schemes (serial powering or DC-DC) to reduce the number of cables.

$>$ Improved cooling system to maintain silicon temperature below $-20^{\circ} \mathrm{C}\left(\mathrm{CO}_{2}\right.$ or $\left.\mathrm{C}_{3} \mathrm{~F}_{8}\right)$.

$>$ Installation: Limited access time inside the cavern. 


\section{Upgrade ID Layout}

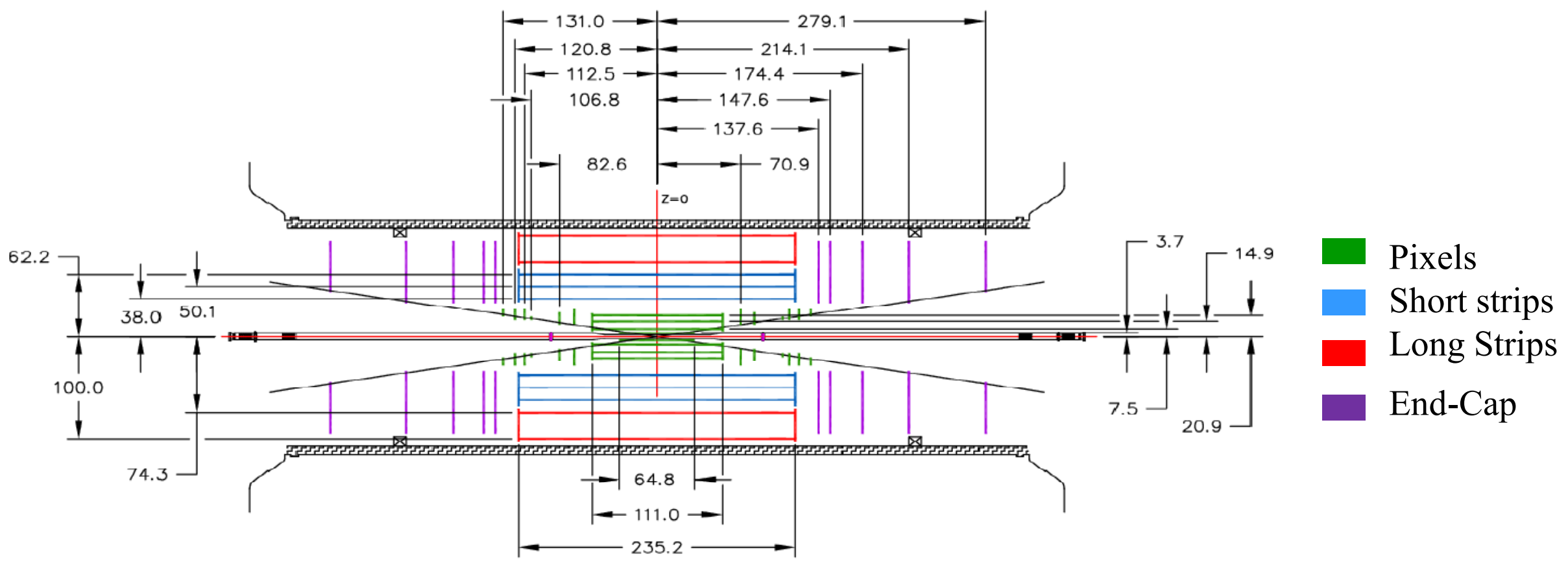

$>$ Pixels: considered options $\rightarrow$ 3-D or diamond detectors for innermost layer and n-on-p or n-on-n detectors for 3 outer layers

> Strips: 5 barrel layers: @ 38, 49,60,75, and 95 cm

- 3 inner layers: SHORT STRIP LAYERS (24 mm-long strips)

- 2 outer layers: LONG STRIP LAYERS (96 mm-long strips)

- 5 double sided disks on each End-Cap

- The 3 outer layers + the end-caps will replace the TRT

- The design is expected to keep the occupancy below $1.6 \%$ at the innermost radius. 


\section{Radiation Hard Technologies: n-on-p}

$>$ p-bulk strip sensors (HPK ATLAS07) are investigated for the ATLAS ID upgrade. Their performance has been evaluated in terms of radiation damage on bulk.

- 6 inch (150 mm) wafers

- $\mathrm{FZ1}<100>(\sim 6.7 \mathrm{k} \Omega \mathrm{cm})$

- $\mathrm{FZ2}<100>(\sim 6.2 \mathrm{k} \Omega \mathrm{cm})$

- P-stop and p-stop+p-spray isolation

- $\quad$ pitch $74.5 \mu \mathrm{m}$, thickness $320 \mu \mathrm{m}$

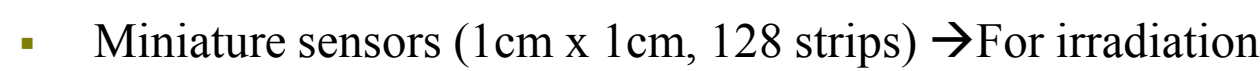
studies

- $\quad$ Full size prototype sensors ( $9.75 \mathrm{~cm}$ x $9.75 \mathrm{~cm}, 1280$ strips $)$

> The sensors are being developed by the R\&D collaboration:

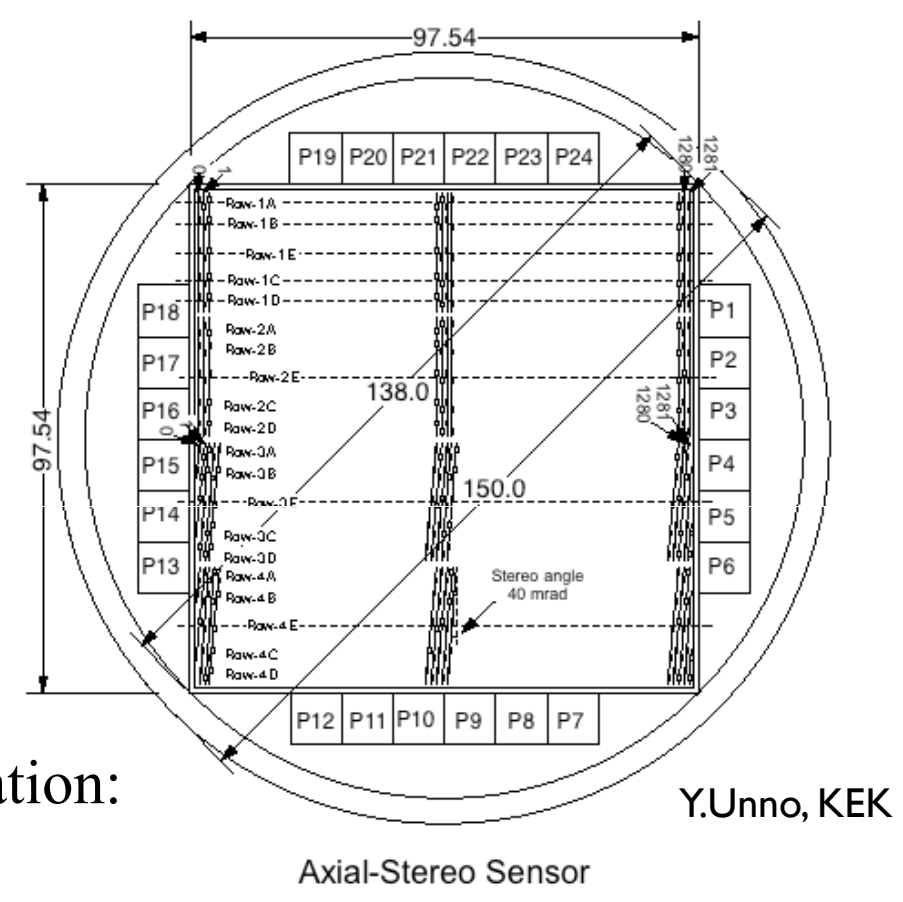

H. Chen, J. Kierstead, J.R. Carter, L.B.A. Hommels, D. Robinson, Univ. of Cambridge

Z. Li, D. Lynn, Brookhaven National Laboratory

K. Jakobs, M. Köhler, U. Parzefall, Physikalisches Institut, Univ. Freiburg

A. Clark, D. Ferrèrre, S. Gonzalez Sevilla, Univ. of Geneva

R. Bates, C. Buttar, L. Eklund, V. O'Shea, Dep. of Physics and Astronomy, Univ. of Glasgow

Y. Unno, S. Terada, Y. Ikegami, T. Kohriki, Institute of Particle and Nuclear Study, KEK

A. Chilingarov, H. Fox, Physics Dep., Lancaster University

A. Affolder, P. P. Allport, H. Brown, G. Casse, A. Greenall, M. Wormald, Oliver Lodge Lab, Univ. of Liverpool

V. Cindro, G. Kramberger, I. Mandić, M. Mikuž, Jožef Stefan Institute and Dep. of Physics, Univ. of Ljubljana

I. Gorelov, M. Hoeferkamp, J. Metcalfe, S. Seidel, K. Toms, Dep. of Physics and Astronomy, Univ. of New Mexico

Z. Dolezal, P. Kodys, Faculty of Mathematics and Physics, Charles Univ. in Prague.

J.Bohm, M.Mikestikova, Academy of Sciences of the Czech Republic

C. Betancourt, N. Dawson, V. Fadeyev, M. Gerling, A. A. Grillo,

S. Lindgren, P. Maddock, F. Martinez-McKinney, H. F.-W. Sadrozinski,

S. Sattari, A. Seiden, J. Von Wilpert, J. Wright, SCIPP, UC Santa Cruz

R. French, S. Paganis, D. Tsionou, Dep. of Physics and Astronomy, The Univ. of Sheffield

B. DeWilde, R. Maunu, D. Puldon, R. McCarthy, D. Schamberger, Dep. of Physics and Astronomy, Stony Brook Univ.

K. Hara, N. Hamasaki, H. Hatano, S. Mitsui, M. Yamada, School of Pure and Applied Sciences, Univ. of Tsukuba

C. García, C. Lacasta, S. Martí i Garcia, M. Miñano, U.Soldevila IFIC (Centro Mixto CSIC-UVEG) 


\section{Radiation Hard Technologies: n-on-p}

\section{Miniature Sensors: Charge Collection}

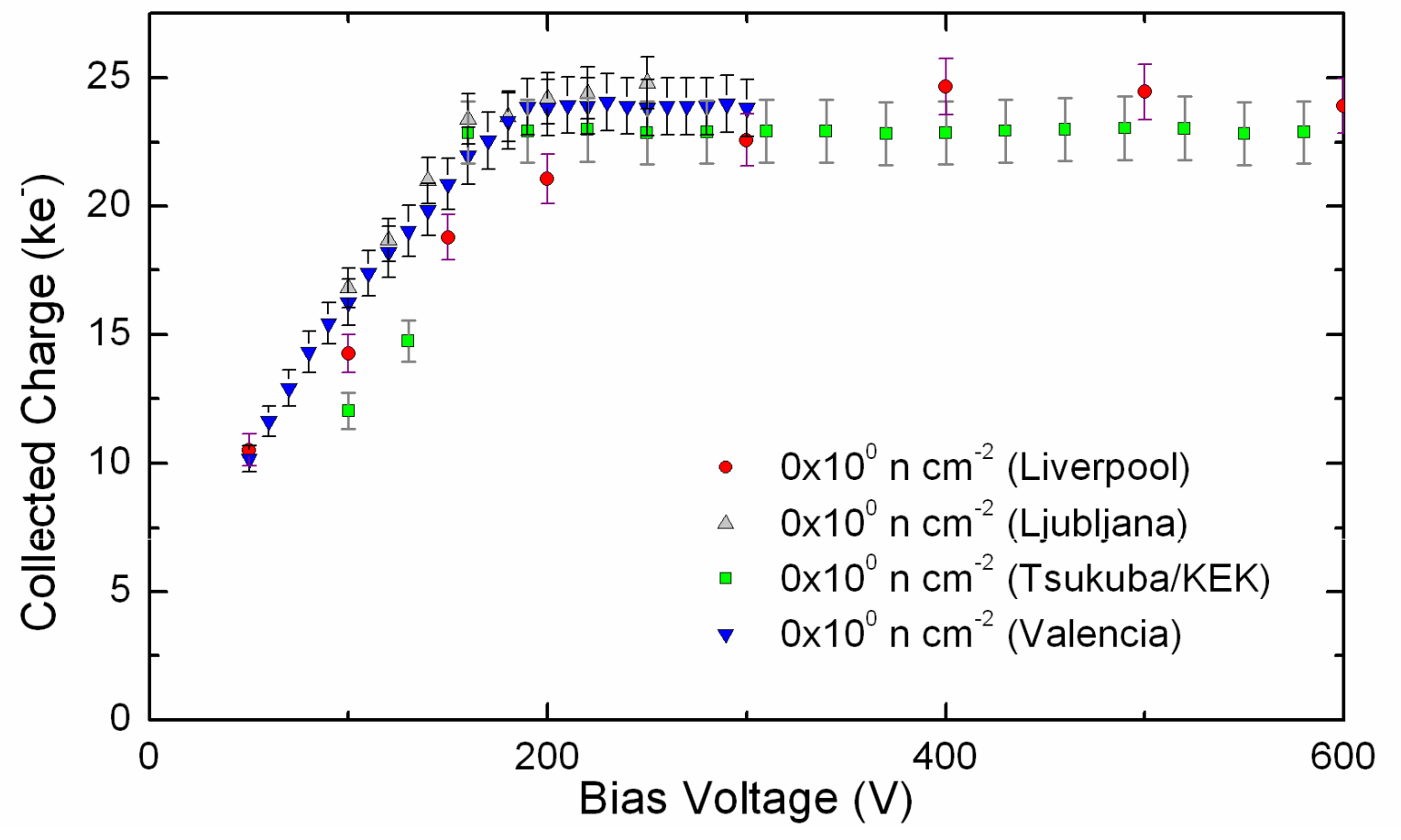

\section{Pre-Irradiation}

Good agreement between sites/systems. Systematic differences under control.

\section{ATLAS institutes involved :}

$>$ Valencia uses Beetle based system (MPV charge, analogue data, 25ns shaping time)

$>$ Ljubljana and Liverpool use SCT128A based system (MPV charge, analogue data, 25ns shaping time)

$>$ Tsukuba/KEK uses a CAMAC 4-ch system with discrete amps (MPV charge, analogue data, 20ns PT)

$>$ UC-Santa Cruz uses PTSM based system (Median charge, binary data, 100 ns shaping time) 


\section{Radiation Hard Technologies: n-on-p}

\section{Miniature Sensors: Neutron Irradiation}
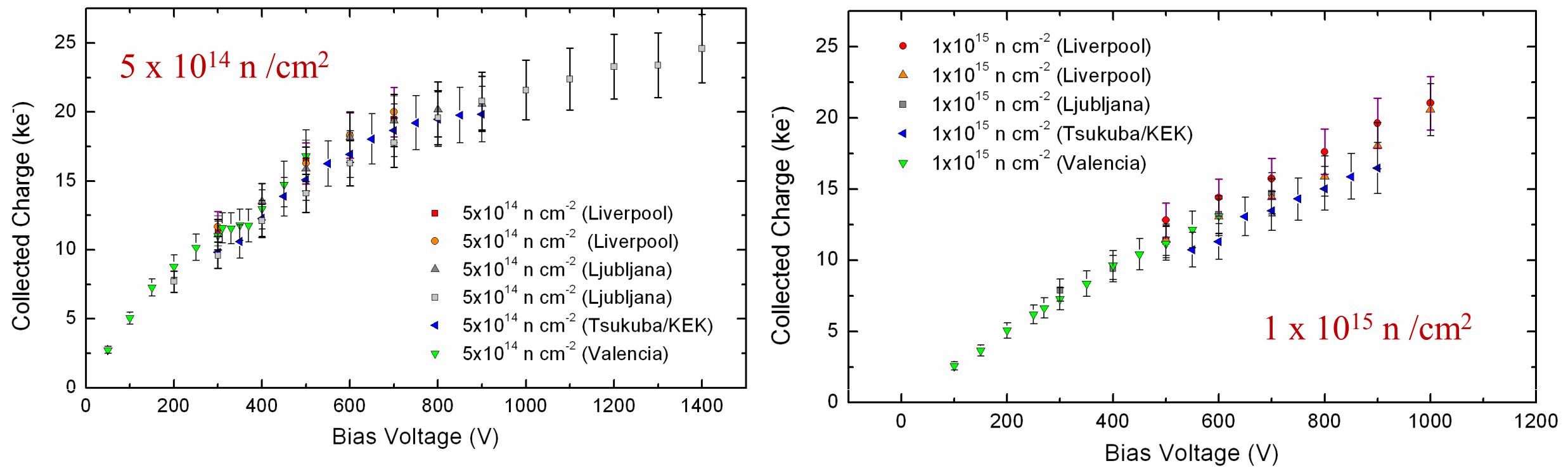

Ljubljana and Tsukuba/KEK annealed for 80 minutes at $60^{\circ} \mathrm{C} \rightarrow$ CCE increases by $\sim 25 \%+/-10 \%$

$>$ Liverpool and Valencia do not anneal (with annealing correction i.e. CCE reduced by $-20 \%+/-10 \%$ )

\begin{tabular}{|c|c|c|c|}
\hline @500V & $\mathbf{C C}$ & Expected noise & S/N achievable \\
\hline @ $5 \times 10^{14} \mathrm{n} / \mathrm{cm}^{2}$ & $14-16 \mathrm{Ke}-$ & $950 \mathrm{e}-$ & $\sim 16$ \\
\hline$@ 1 \times 10^{15} \mathrm{n} / \mathrm{cm}^{2}$ & $11-13 \mathrm{Ke}-$ & $600 \mathrm{e}-$ & $\sim 20$ Good! \\
\hline
\end{tabular}




\section{Radiation Hard Technologies: n-on-p}

\section{Miniature Sensors: Proton Irradiation}
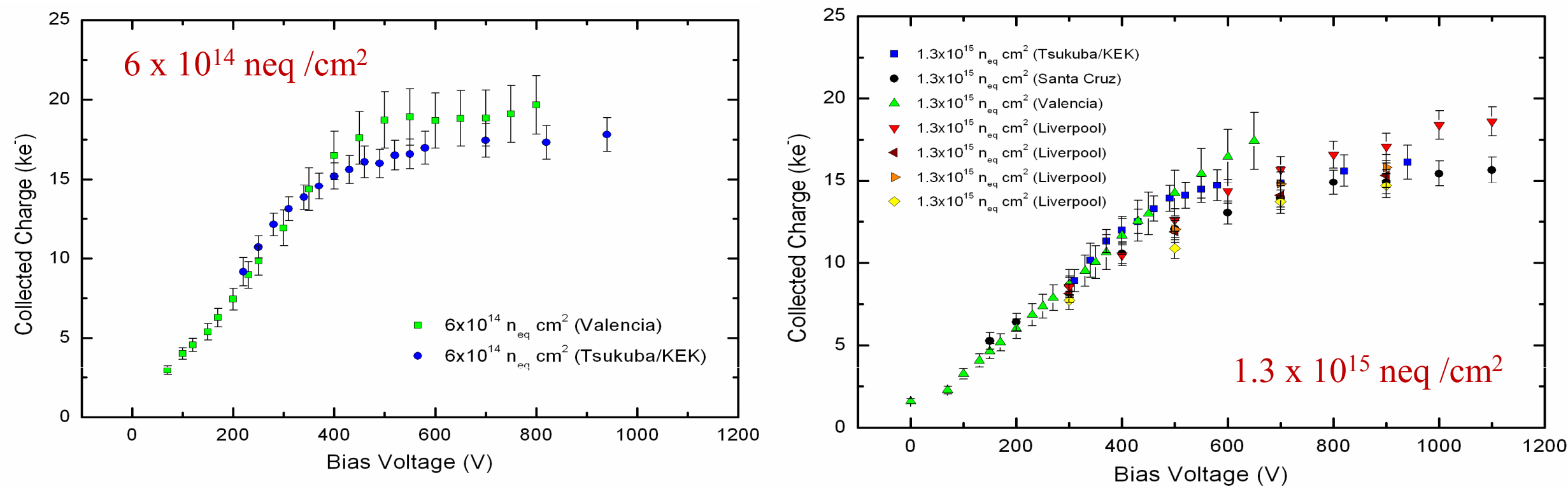

Ljubljana and Tsukuba/KEK annealed for 80 minutes at $60^{\circ} \mathrm{C} \rightarrow \mathrm{CCE}$ increases by $\sim 25 \%+/-10 \%$

$>$ Liverpool and Valencia do not anneal (with annealing correction i.e. CCE reduced by $-20 \%+/-10 \%$ )

\begin{tabular}{|c|c|c|c|}
\hline @500V & CC & Expected noise & \multicolumn{2}{|c|}{ S/N achievable } \\
\hline$@ 5 \times 10^{14} \mathrm{n} / \mathrm{cm}^{2}$ & $16-19 \mathrm{Ke}-$ & $950 \mathrm{e}-$ & $\sim 18$ Good! \\
\hline$@ 1 \times 10^{15} \mathrm{n} / \mathrm{cm}^{2}$ & $11-14 \mathrm{Ke}-$ & $600 \mathrm{e}-$ & $\sim 20$ \\
\hline
\end{tabular}




\section{Radiation Hard Technologies: n-on-p}

\section{Miniature Sensors: N-on-P FZ Irradiations (HPK and Micron sensors)}

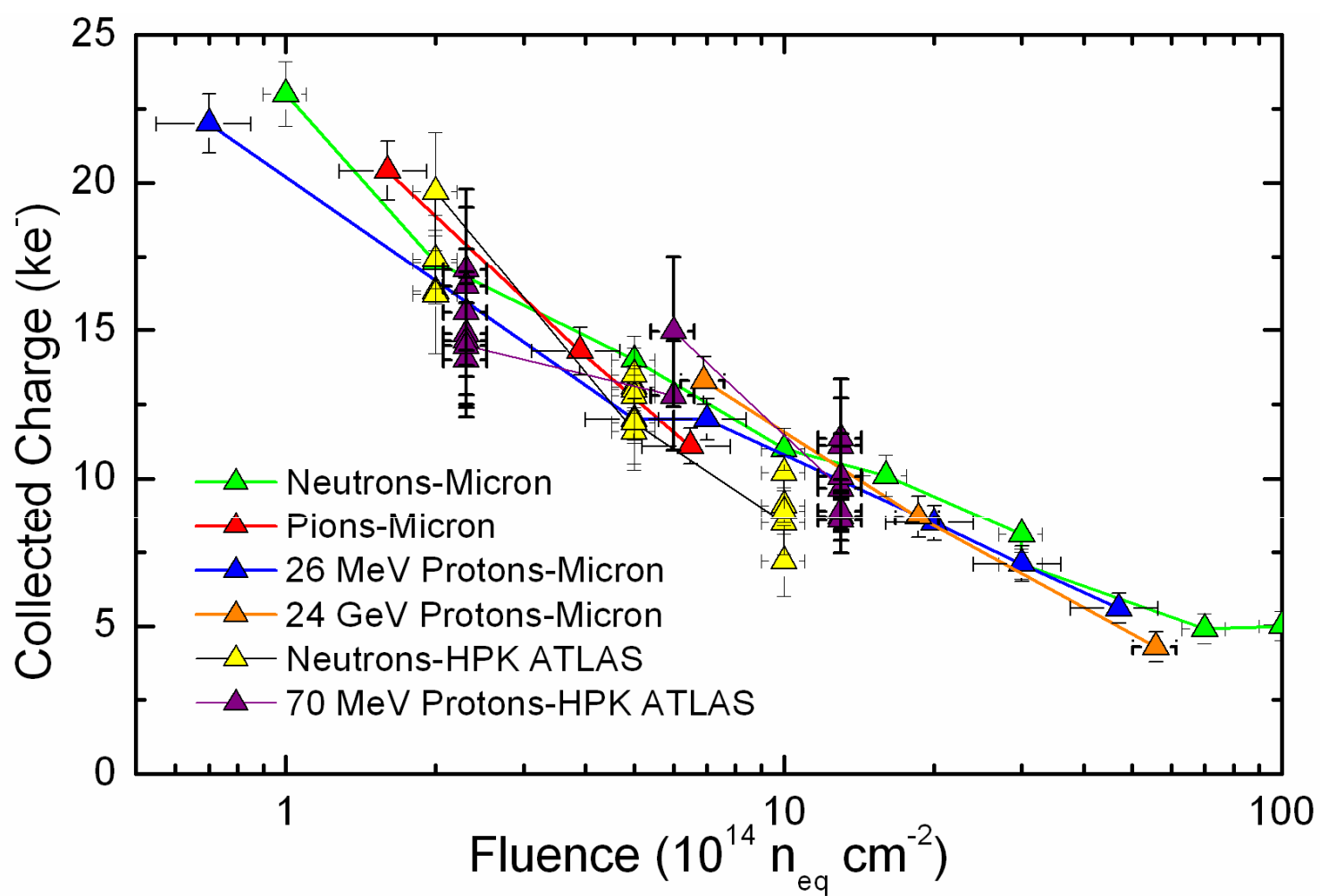

Micron data from

A. Affolder, P.P.Allport \& G. Casse, to be published in the proceedings of TIPP09.

$>$ Performance of n-on-p FZ sensors produced at Micron and Hamamatsu are the same after all measured irradiation sources. HPK data shown from all sites. Pion irradiation measurements corrected for annealing during run. 


\section{Radiation Hard Technologies: n-on-p}

\section{Miniature Sensors: Full Depletion Voltage Evaluation}

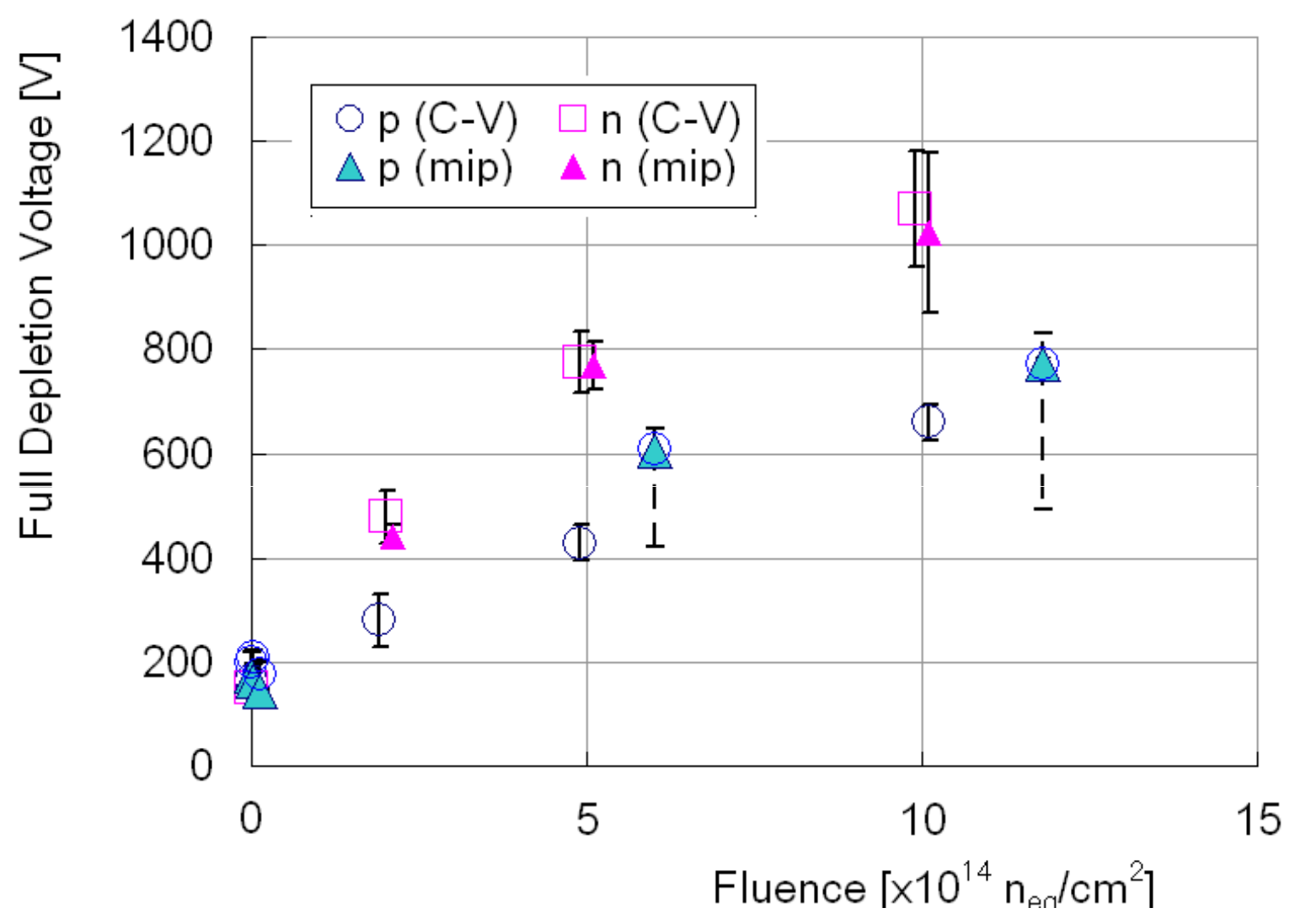

\section{$\underline{\text { Series Sensors FZ1 }}$}

Protons:

FDV 700V@1015 $\mathrm{cm}^{-2}$

$>$ Neutrons:

FDV $\sim 800 \mathrm{~V}$

(a) $5 \times 10^{14} \mathrm{~cm}^{-2}$

K. Hara et al., "Testing of bulk radiation damage of n-in-p silicon sensor for very high radiation environment" $7^{\text {th }}$ International "Hiroshima" Symposium on the Development and Applications of Semiconductor Tracking Detectors. 


\section{Radiation Hard Technologies: n-on-p}

\section{Full Size Prototype Sensors: Electrical Characterization}

ATLAS institutes involved:

$>$ University of Cambridge

$\longrightarrow 2$ sensors: W15, W16

$>$ Stony Brook University $\longrightarrow 9$ sensors: W19, W21-23, W25-29

$>$ Inst. of Physics and Charles Univ, Prague $\longrightarrow 6$ sensors: W32, W33, W35, W37, W38, W39

$>$ University of Geneva 2 sensors: $\mathrm{W} 17, \mathrm{~W} 18$

Total number of tested sensors: 19

ATLAS07 Full Size Sensors

$>9.75 \mathrm{~cm} \times 9.75 \mathrm{~cm}$

$>4$ segments:

- two with "axial" strips. $74.5 \mu \mathrm{m}$ pitch

- two with "stereo" strips. $74.4 \mu \mathrm{m}$ pitch, $40 \mathrm{mrad}$

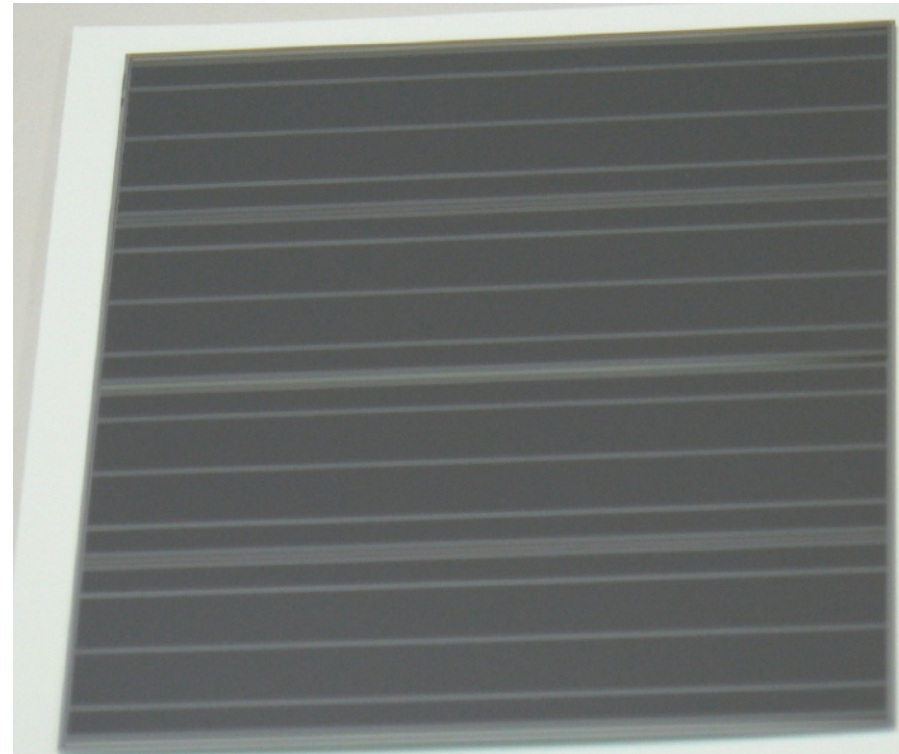




\section{Radiation Hard Technologies: n-on-p}

\section{Full Size Prototype Sensors: Bias Scan}

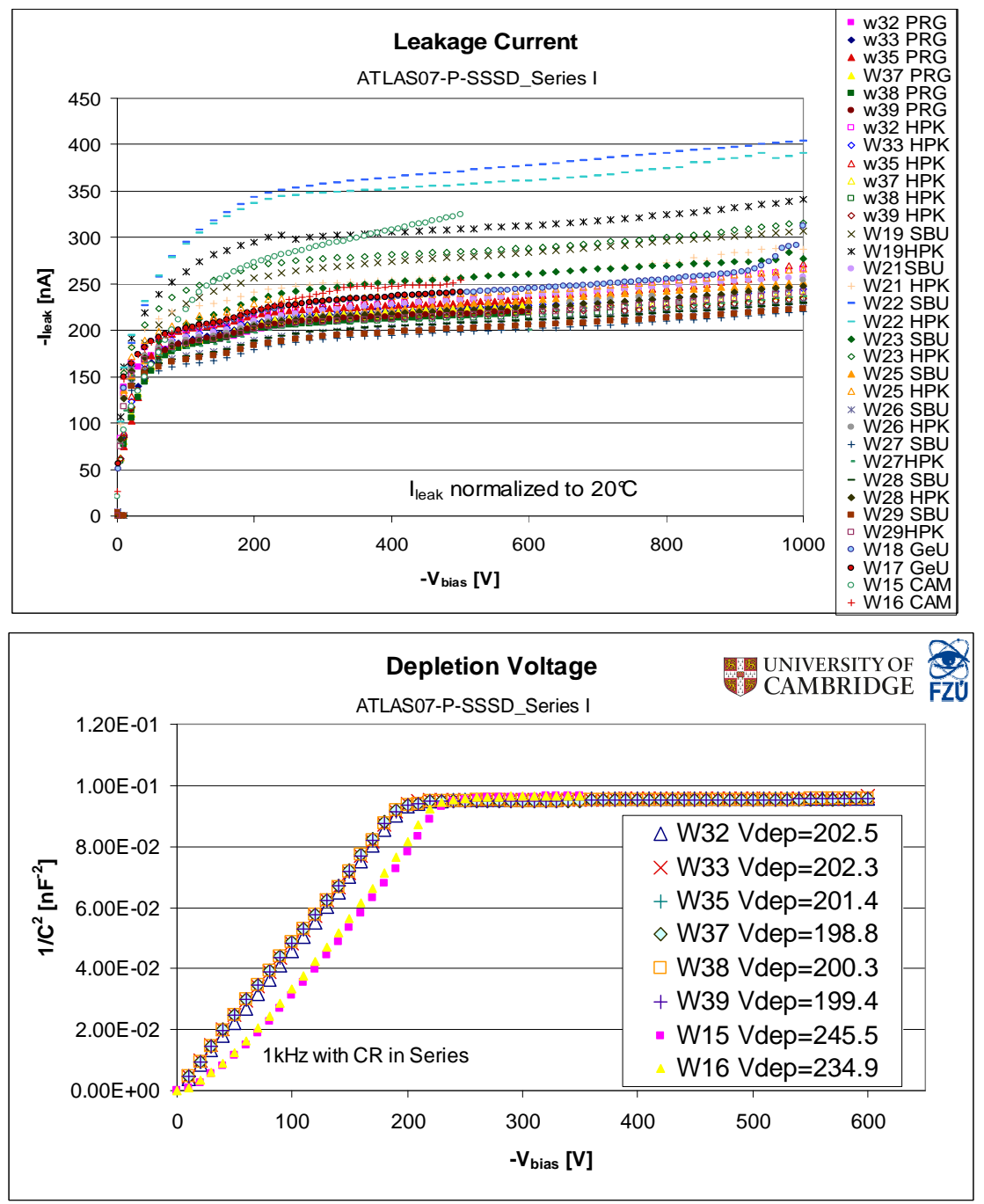

$>$ No microdischarges with exception one sensor (Vbd 420V).

$>$ Sensors satisfy the ATLAS07 Technical Specification (<200um@600V)

$>$ IV scan was repeated after bias scan and strip scan.

$>$ Current was usually higher by $10 \%-20 \%$ and breakdown for 2 sensors at $\sim 380 \mathrm{~V}$.

Estimated values of Vdep:

- 6 sensors (Prague) 199-203V

- 2 sensors (Cambridge) 235-245V

$>$ All tested sensors satisfy specifications:

$$
\text { Vdep }<500 \mathrm{~V}
$$




\section{Radiation Hard Technologies: n-on-p}

\section{Full Size Prototype Sensors: Bias Scan}

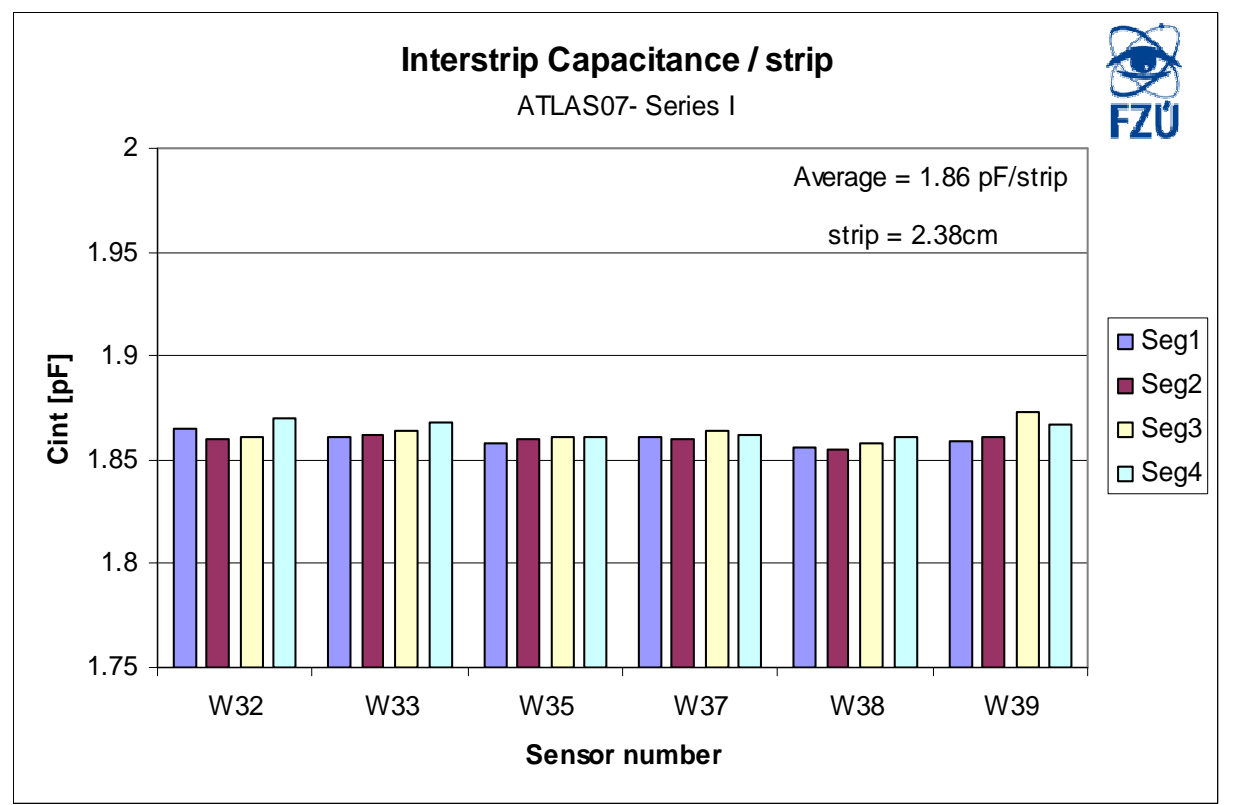

All tested sensors:

Cint $\sim 0.75-0.80 \mathrm{pF} / \mathrm{cm}$

$<1.1 \mathrm{pF} / \mathrm{cm}$ (ATLAS specification)

Cint $/$ strip $=1.86 \mathrm{pF} / \mathrm{strip}$

$>$ Measurements taken on central strip with either neighbour grounded. Including next-to-neighbours results in $10-15 \%$ higher readings. 


\section{Radiation Hard Technologies: n-on-p}

\section{Full Size Prototype Sensors: Strip Scan}

For 5 sensors
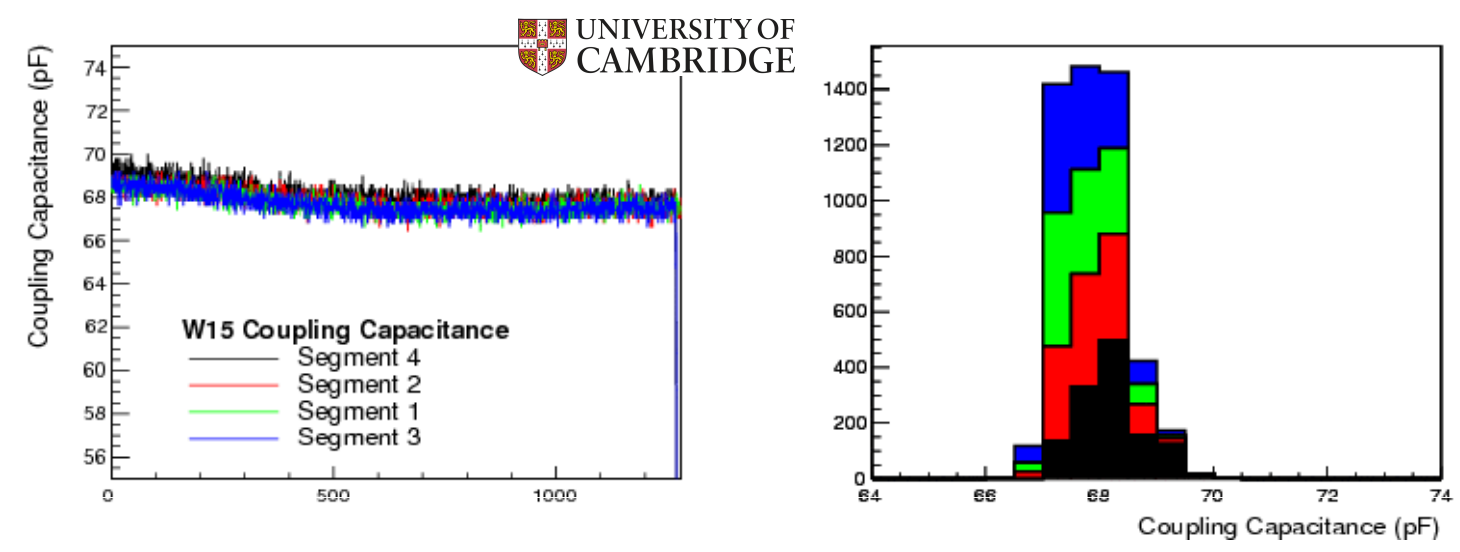

$\mathrm{Ccpl}=66-68 \mathrm{pF} / \mathrm{strip}$

ATLAS07 Specification:

$\mathrm{Ccpl} / \mathrm{strip}>47.6 \mathrm{pF}$

Strip length $=2.38 \mathrm{~cm}$

Rbias $=1.30 \mathrm{M} \Omega-1.45 \mathrm{M} \Omega$

ATLAS07 Specification:

Rbias $=1.5 \pm 0.5 \mathrm{M} \Omega$ 


\section{Module Integration Concepts}

Barrel Stave $>$ Hybrid glued to sensors. These glued to bus tape. This glued to cooling substrate.

$60 \mathrm{~cm}, 9 \mathrm{~cm}$ strip, 4 chips wide

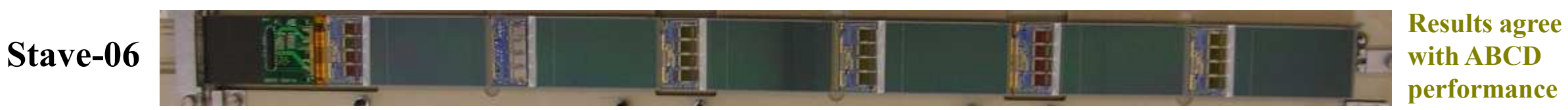

$1 \mathrm{~m}, 3 \mathrm{~cm}$ strip, 6 chips wide

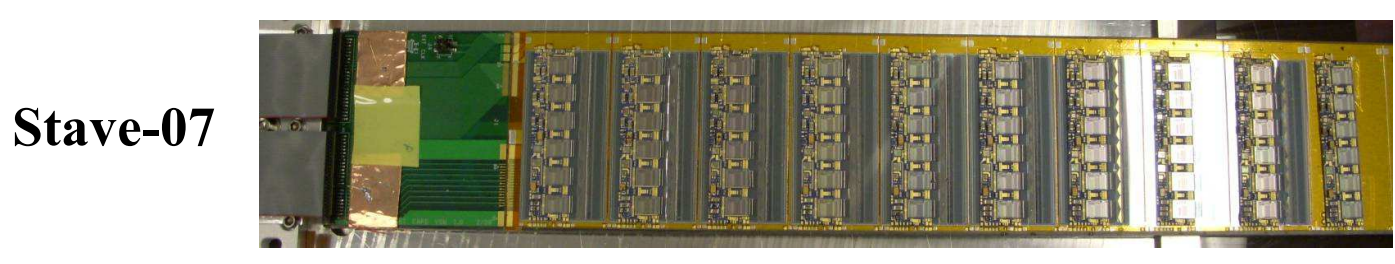

Strip Stave from $L B N L$

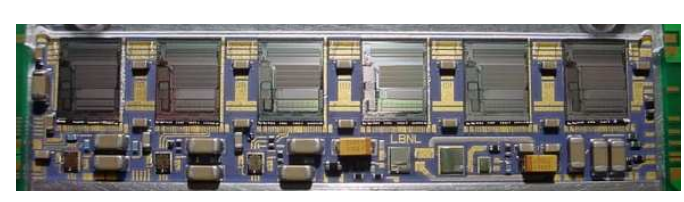

- Individual hybrids/modules work well electrically. Good noise performance. All are 900e-

- Tested 6 module on stave with ABCD chips. Serial Powering lines.

-Working ongoing

Under Construction: $1.2 \mathrm{~m}, 2.5 \mathrm{~cm}$ strip, 10 chips wide (20 chips/hybrid)

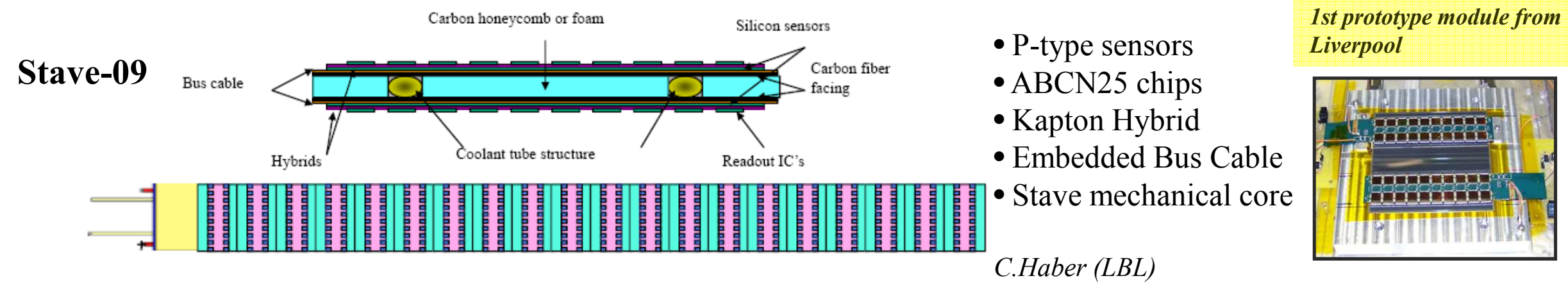




\section{Module Integration Concepts}

\section{Petal $>$ Follows quite closely the barrel stave concept}

\section{C.Lacasta (IFIC, Valencia)}

$>2$ carbon facings + Honeycomb sandwich core

$>$ Independent e-services + Bus Cable

$>$ Independent $\mathrm{C} 02$ cooling pipe

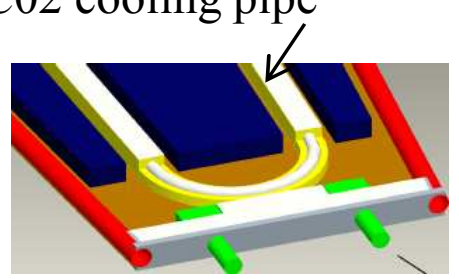

$>$ petal surface: $830 \mathrm{~cm}^{2}$

$>5$ disks on each end-cap

$>32$ petals/disk (16 on each side)

$>6$ different detector types mounted on petal

$>9$ different hybrid types

$>116$ chips/petal
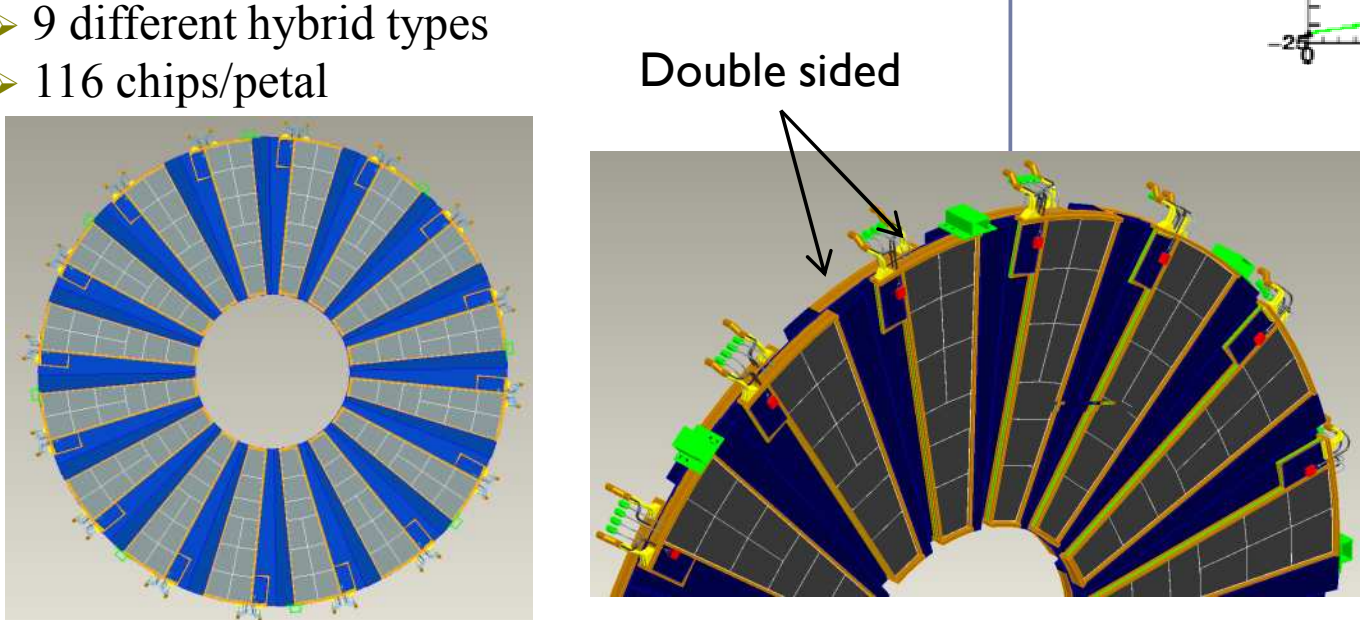

Simulations of a disk. Issues: Layout, modularity, powering

\section{Thermal simulations to explore the behaviour at critical points:}

$>$ Assumed $-30^{\circ} \mathrm{C}$ coolant temperature $\left(-27^{\circ} \mathrm{C}\right.$ on the return pipe)

$>$ The simulation results show that the temperature on sensors is within safety range (to be confirmed with prototypes) .
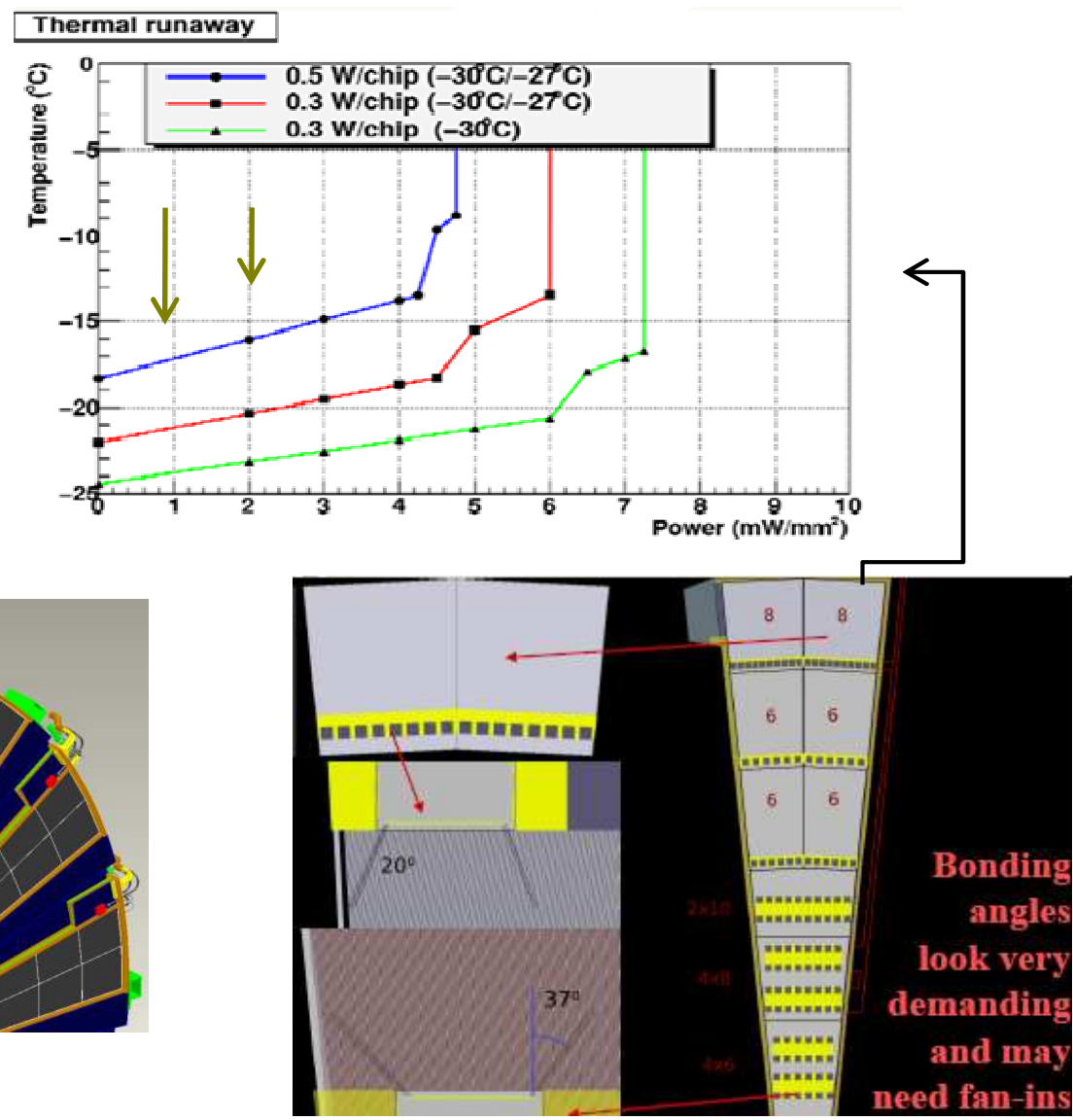


\section{Module Integration Concepts}

\section{Super-Module}

1) Build individual modules :

$>$ Double sided module

$>2$ silicon (short) microstrip sensors: $\mathrm{n}+$-on-p, $10 \times 10 \mathrm{~cm}^{2}$

$>4$ bridged hybrids with $\mathrm{ABCN}$ asics each

2) Insert modules into a frame: Super-module (Based on SCT experience) 2 proposals for module integration into cylinders:

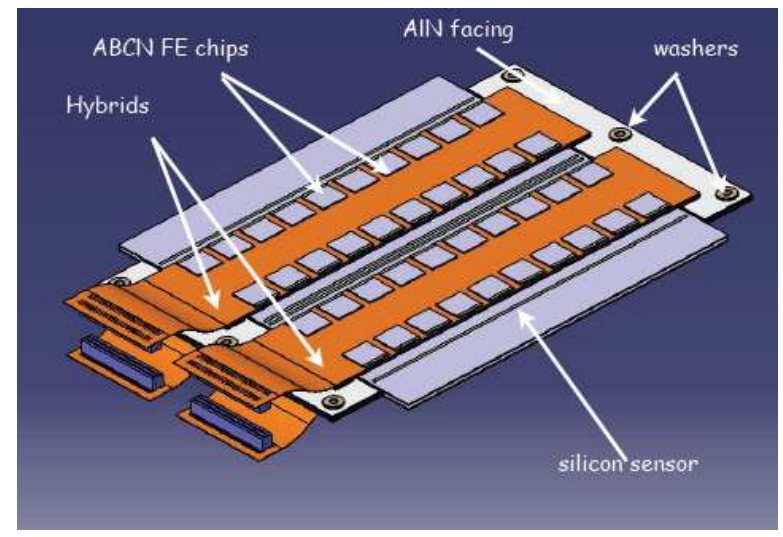

Lateral insertion (KEK): Installation of the Super-Modules, cylinder by cylinder

$>$ End-insertion (Geneva): Barrel structures can be assembled before the Super-Modules are integrated.
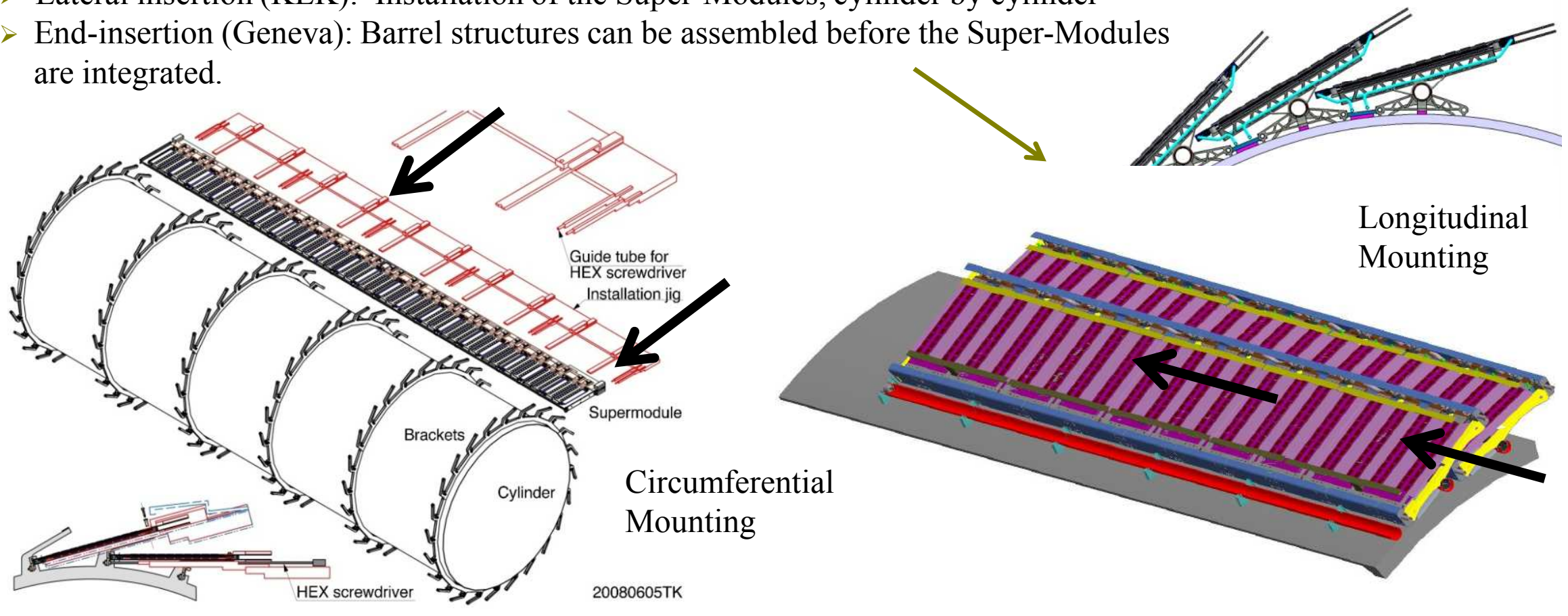

Longitudinal Mounting 


\section{Conclusions}

$>$ The tracker of ATLAS will have to be replaced for the LHC upgrade.

$>$ Lots of R\&D has already been carried out and ideas are near to converge.

$>$ Strip community are investigating the short $(2.4 \mathrm{~cm})$ and the long strips $(9.6 \mathrm{~cm})$ sensors for barrel and EC with stave or petal concept.

$>$ Miniature and Full Size p-type sensors have been manufactured by Hamamatsu (HPK):

- Good performance in terms of charge collection efficiency under neutron and proton irradiation. S/N of $\sim 20$ (16) should be achievable with short (long) strip detector.

- Sensors will operate at partial depletion $\rightarrow$ p-bulk sensor good candidate.

- All tested full size sensors satisfy ATLAS07 Technical Specification for leakage current, full depletion voltages, Cint measured in the bias voltage scan.

- Strip scan was performed on 5 full size sensors. Ccoupling and Rbias were uniform across the whole sensor and within specifications.

$>$ Prototyping for Module Integration is progressing.

> Good progress but important decisions to take.

- Thank you - 
- Backup - 


\section{Backup}

$>$ Services:

- The required high granularity supposes (in the same space)

- x5 number of channels

- $\mathrm{x} 5$ number of cables<smiles>C=[14CH]</smiles>

New options in powering (not individual powering)

\begin{tabular}{|c|c|c|c|c|c|}
\hline Current ID & Area $\left(\mathbf{m}^{2}\right)$ & Channels & Upgrade & Area $\left(\mathbf{m}^{2}\right)$ & Channels \\
\hline Pixels & 1.8 & $80 \mathrm{M}$ & Pixels & 5 & $\sim 300 \mathrm{M}$ \\
\hline SCT & 61 & $\sim 6.3 \mathrm{M}$ & Short Strips & 60 & $\sim 30 \mathrm{M}$ \\
\hline TRT & & $400 \mathrm{~K}$ & Long Strips & 100 & $\sim 15 \mathrm{M}$ \\
\hline
\end{tabular}




\section{Backup}

- Several options on powering: DC/DC or serial.

- Cannot have individual module powering $\rightarrow$ too much material and no space.

- Requirements: High power efficiency, low noise, safety (overcurrent, overvoltage, overtemperature).

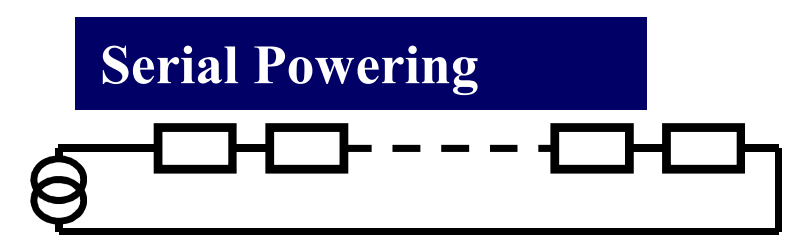

\section{DC-DC Powering}

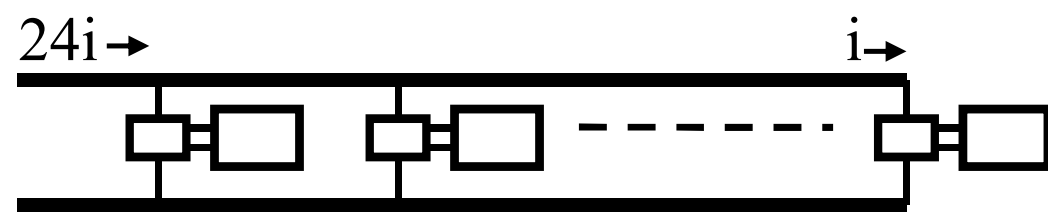

Serial Powering scheme has been shown to perform well on 6 and 30 module staves (Stave06,Stave07)

- Excellent noise performances

- Current issues:

- Protection schemes (shunt regulators) possible integration into FE chips

- Custom current source

DC/DC scheme:

- Only 1 power line/stave (10-12V)

- Distribution with 2 conversion stages:

- Stage $1 \rightarrow 2$ converters: $2.5 \mathrm{~V}$ analog and $1.8 \mathrm{~V}$ digital

- Stage $2 \rightarrow$ On-chip switched capacitor

- High granularity of the power distribution

-Very flexible 


\section{Backup}

\section{Schedule}

Currently anticipated evolution of the ATLAS upgrade (http://atlas.web.cern.ch/Atlas/GROUPS/UPGRADES/)

\begin{tabular}{|c|c|}
\hline Milestone & Date \\
\hline Straw Man \& options fixed & Dec 2006 \\
\hline R\&D towards inner detector conceptual design & $2007-2010$ \\
\hline LoI & May 2010 \\
\hline Technical Proposal, Initial MoU and Costing & April 2012 \\
\hline Inner Tracker TDR & Dec 2013 \\
\hline $\begin{array}{c}\text { Production readiness reviews and ramp up } \\
\text { production }\end{array}$ & 2014 \\
\hline New Insertable B-layer Installation & End 2014 \\
\hline Procure parts, Component assembly & $2014-2016$ \\
\hline Surface assembly & September 2016 - end 2017 \\
\hline Surface testing & 2018 \\
\hline Stop LHC & Sep 2018 \\
\hline Remove old detectors, install new & Oct 2018 - Dec 2019 \\
\hline Commission new detectors & Jan 2020 - Mar 2020 \\
\hline Take data & April 2020 \\
\hline
\end{tabular}




\section{Backup}

\section{Current Inner Detector}

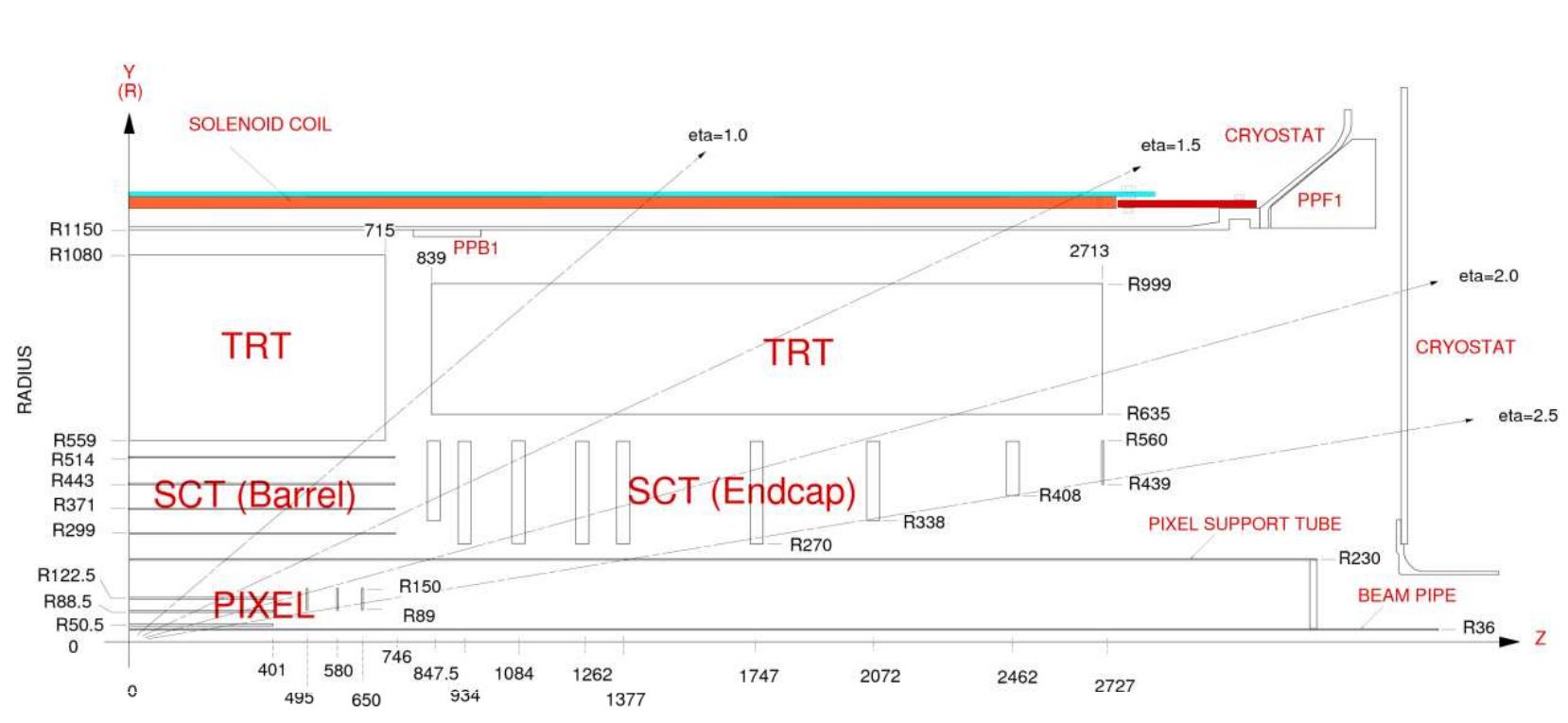

Pixels ( $\mathrm{n}+$-on- $\mathrm{n}$ sensor technology): 3 barrels $+2 \times 3$ discs $(5<\mathrm{R}<15 \mathrm{~cm})$

Strips (SCT) (p+-on-n sensor technology ): 4 barrels $+2 x 9$ discs $(30<\mathrm{R}<51 \mathrm{~cm})$

TRT: Barrel + Wheels ( $4 \mathrm{~mm}$ diameter straw drift tubes) $(55<\mathrm{R}<105 \mathrm{~cm})$

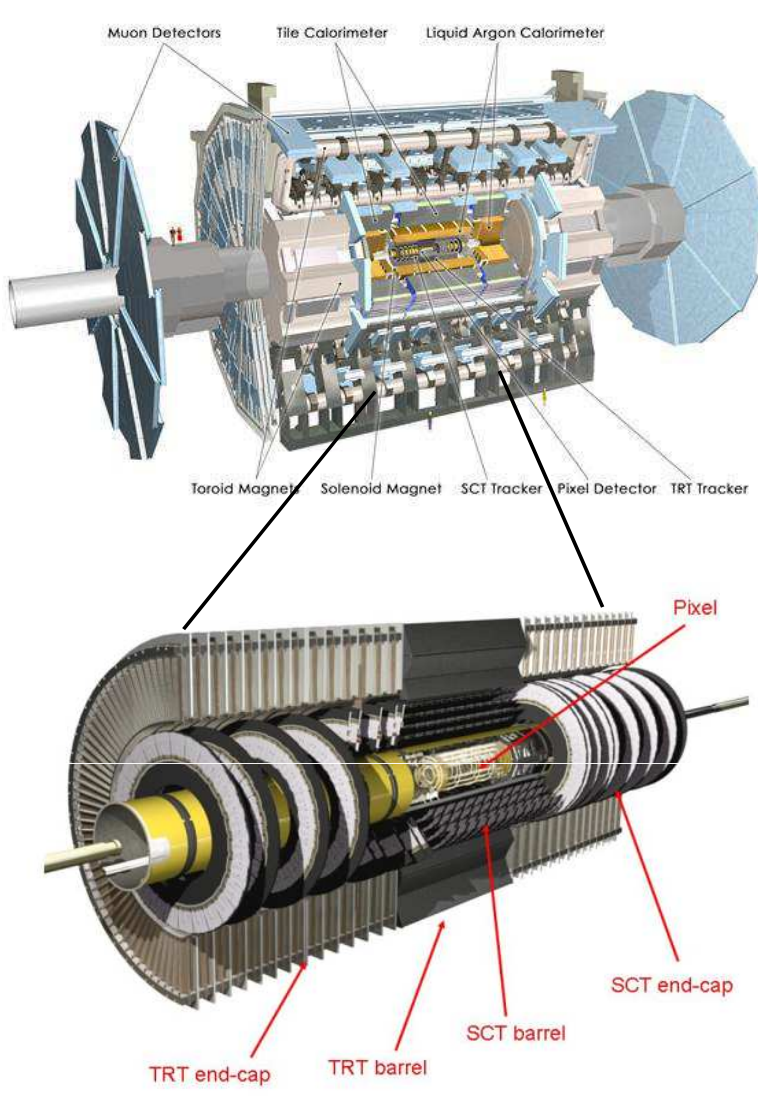

Designed fluences for sensors:

- Pixel layer 0: 1 x $10^{15} 1 \mathrm{MeV}$ n-equivalent $/ \mathrm{cm}^{2}$

- SCT Barrel layer 1: $8 \times 10^{14} 1 \mathrm{MeV}$ n-equivalent $/ \mathrm{cm}^{2}$

- SCT End-cap disc 9: 7 x 10 $141 \mathrm{MeV}$ n-equivalent $/ \mathrm{cm}^{2}$

- TRT outer radius: $3 \times 10^{13} 1 \mathrm{MeV}$ n-equivalent $/ \mathrm{cm}^{2}$
SCT Module (Barrel \& Endcaps)Designs
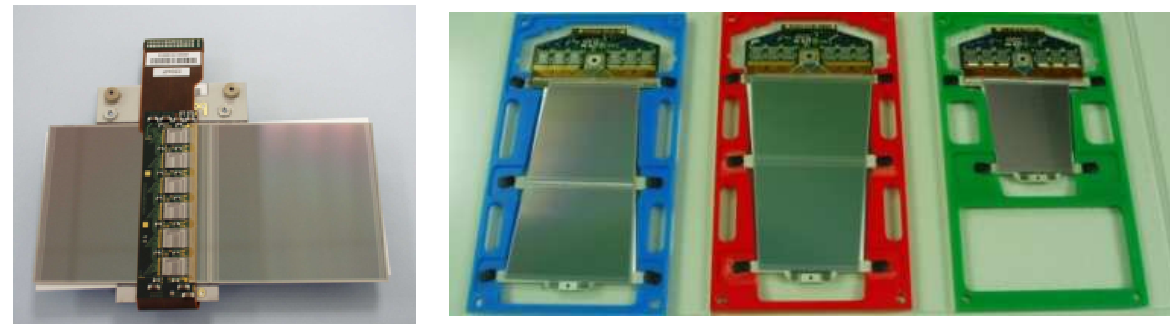


\section{Backup}

\section{p-type Detectors: Motivation}

The current SCT sensors use p-on-n technology. They are not sufficient radiation hard for the LHC upgrade.
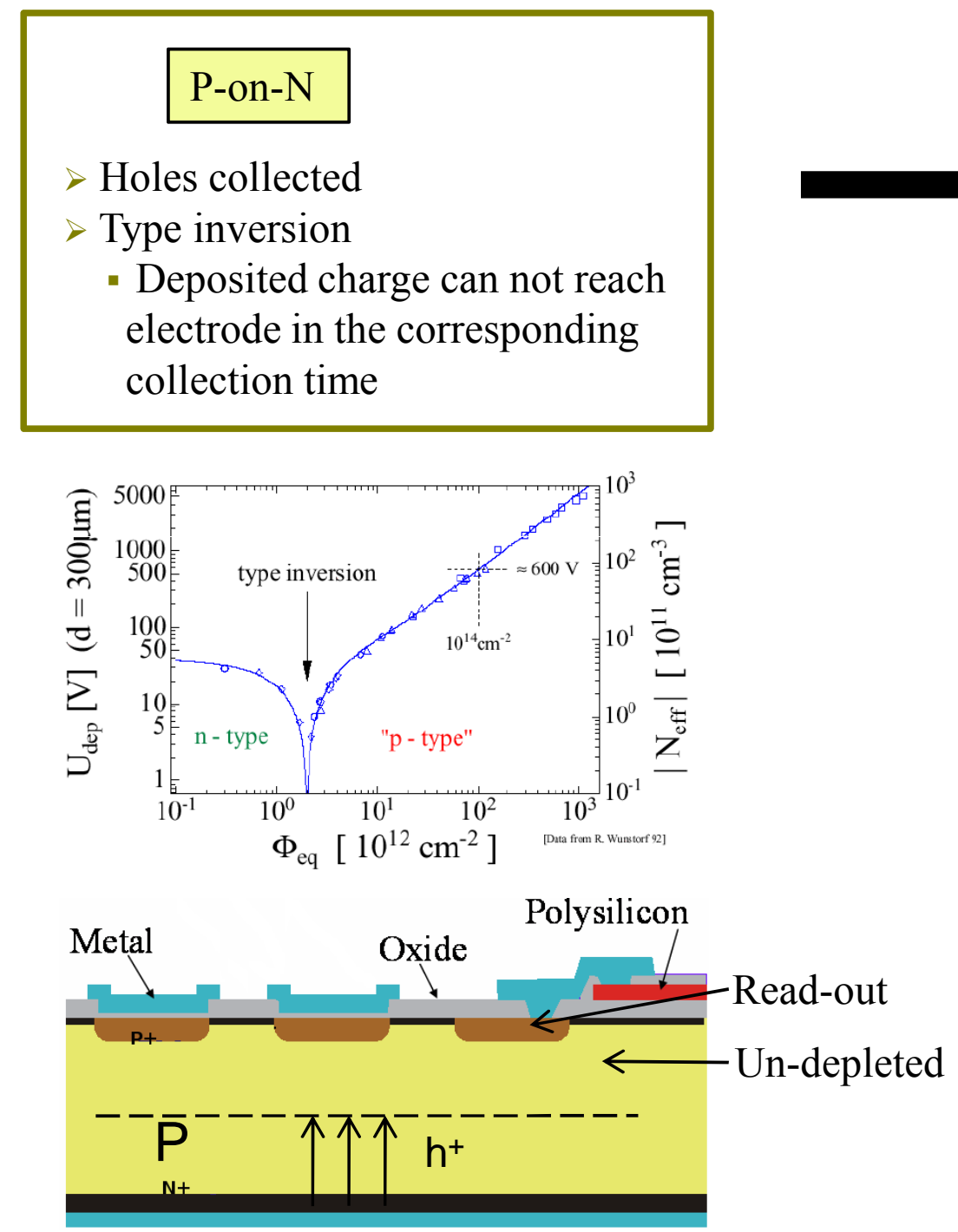
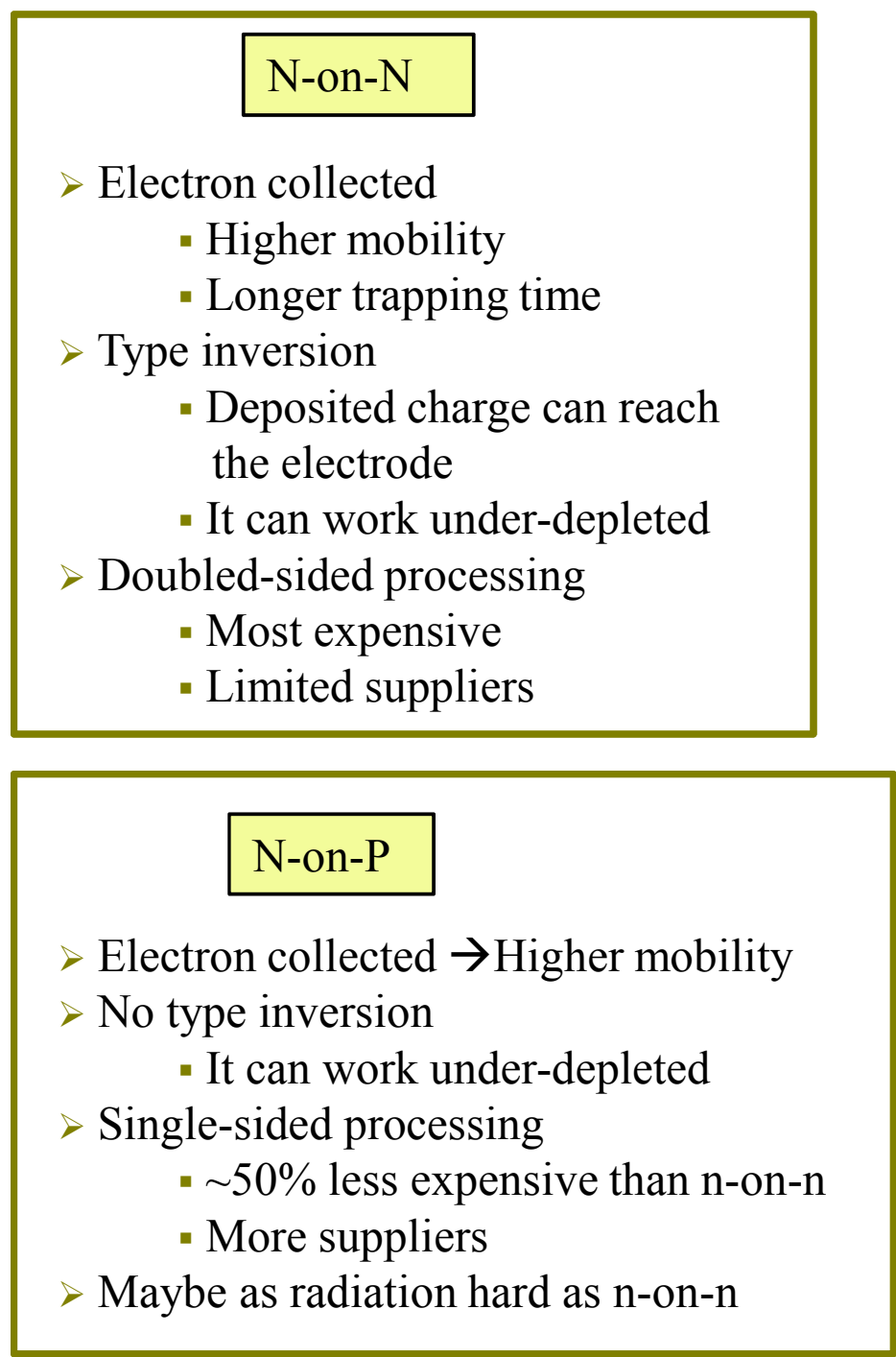


\section{Current SCT Detector}

\section{Strips Detector in numbers}

\begin{tabular}{|c|c|c|c|c|c|c|}
\hline \multirow{9}{*}{ Barrel } & Layer & Type & $\begin{array}{l}\text { Radius } \\
\text { [cm] }\end{array}$ & $\begin{array}{c}\text { Phi } \\
\text { segmentation }\end{array}$ & \begin{tabular}{|c|} 
Number of \\
modules per half \\
single sided \\
stave
\end{tabular} & $\begin{array}{c}\text { Number of 128-ch } \\
\text { FEC per half single } \\
\text { sided stave }\end{array}$ \\
\hline & 0 & Short Strips & 38 & 28 & 10 & 400 \\
\hline & 1 & Short Strips & 49 & 36 & 10 & 400 \\
\hline & 2 & Short Strips & 60 & 44 & 10 & 400 \\
\hline & 3 & Long Strips & 75 & 56 & 19 & 190 \\
\hline & 4 & Long Strips & 95 & 72 & 19 & 190 \\
\hline & \multicolumn{5}{|c|}{ Total number of staves for the Barrel } & 236 \\
\hline & \multicolumn{5}{|c|}{ Total number of modules for the Barrel } & 14,336 \\
\hline & \multicolumn{5}{|c|}{ Total number of $\boxplus$ (ffor the Barrel } & 270,080 \\
\hline \multirow{2}{*}{ Endcap } & \multicolumn{5}{|c|}{ Total number of staves for one End-cap } & 1,152 \\
\hline & \multicolumn{5}{|c|}{ Total number of 128-ch \#Cfor the two End-cap } & 57,088 \\
\hline \multicolumn{6}{|c|}{ Total number of 128-channel F日CS } & 327,168 \\
\hline \multicolumn{6}{|c|}{ Total amount of channels } & $41,877,504$ \\
\hline
\end{tabular}

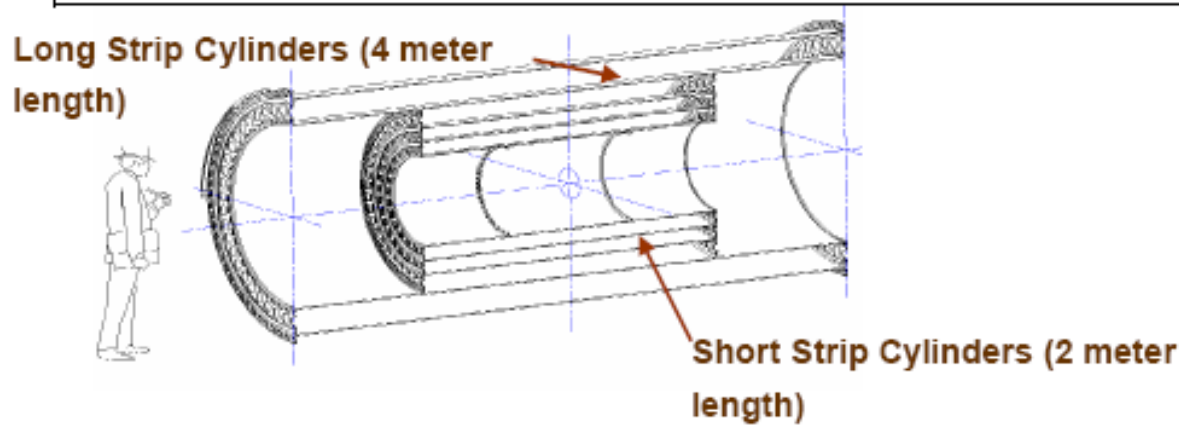

- Current SCT detector

- 4088 modules

- 49k 128-channel FEIC

- 6.3M channels

Philippe Farthouat (CERN) 


\section{Backup}

\section{Leakage Current (@500/800V) with protons}

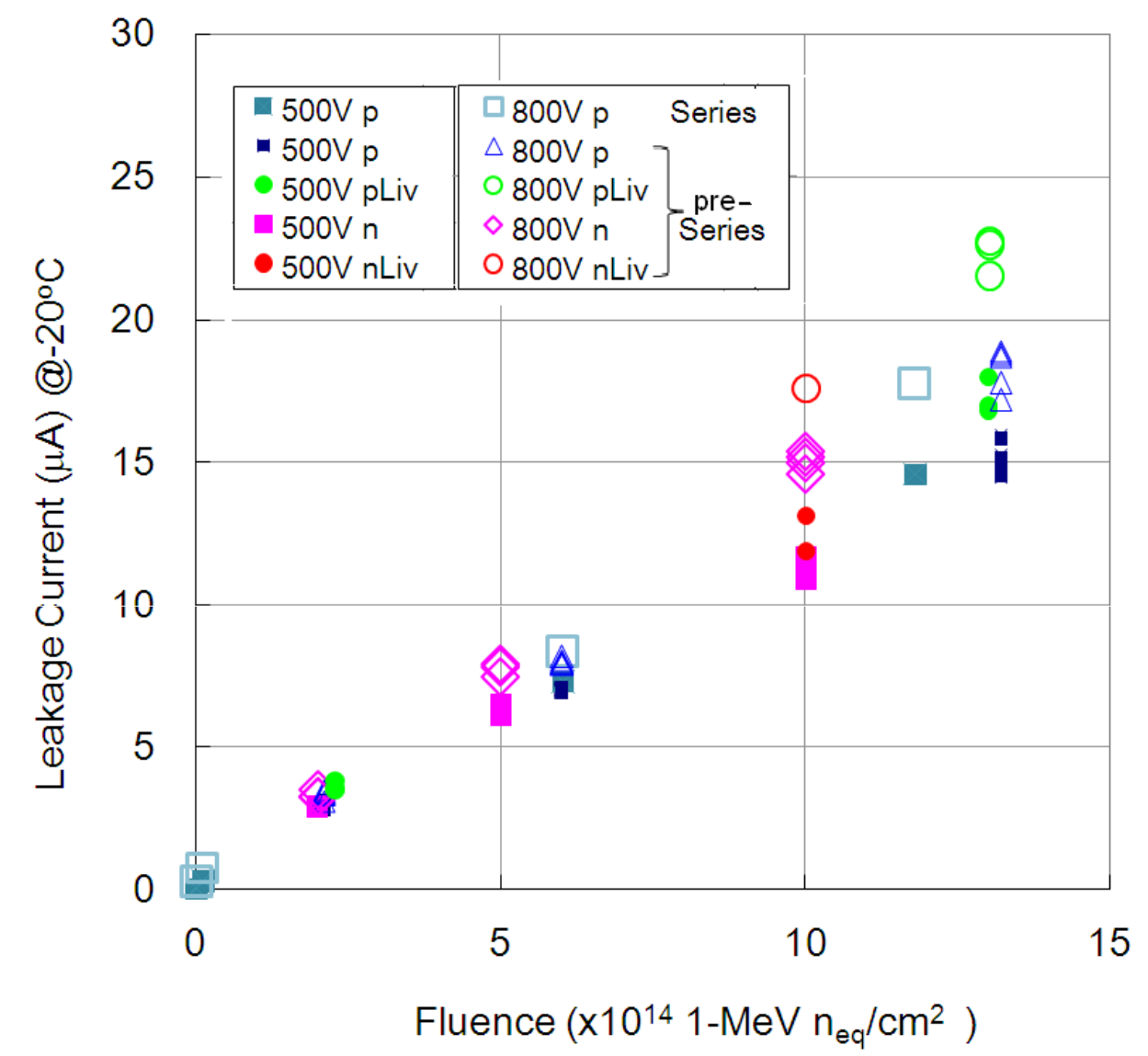

Current $/ \mathrm{V}=\alpha \cdot \phi$

$\mathrm{V}_{\mathrm{FD}} \sim 700 \mathrm{~V}$

$\phi=10^{15} \mathrm{~cm}^{-2}$

$>$ The damage constant (slope)

$\sim$ consistent with n-bulk damage constant $\left(\alpha \sim 4 \times 10^{-17} \mathrm{~A} / \mathrm{cm}\right)$

$>$ The leakage current of non-irradiated pbulk sensors is at the similar level to HPK n-bulk sensors

\section{Agreement between sites \\ (Tsukuba/KEK and Liverpool)}

\section{$>$ proton/neutron damages contribute} similarly to leakage current increase

K. Hara et al., "Testing of bulk radiation damage of n-in-p silicon sensor for very high radiation environment", $7^{\text {th }}$ International "Hiroshima" Symposium on the Development and Applications of Semiconductor Tracking Detectors. 